\section{Hipertensión arterial pulmonar: el entrenamiento físico como complemento de la terapia farmacológica}

\author{
ASTRID VON OETINGER ${ }^{1,2, a}$, LUZ MARÍA TRUJILLO ${ }^{3,4, a}$, \\ SERGIO VILLANUEVA ${ }^{5, b, c}$, MÓNICA ZAGOLIN ${ }^{6,7,8}$
}

\section{Cardiopulmonary rehabilitation in pulmonary arterial hypertension}

Cardiopulmonary rehabilitation is a promising therapy for Pulmonary arterial hypertension (PAH) whose survival does not exceed $65 \%$ at five years. We performed a literature search about rehabilitation on PAH in MEDLINE, LILACS and COCHRANE databases, considering articles from 2005 to 2017. Fifteen articles were incorporated in the final analysis. We obtained information about safety parameters, type of exercises applied, duration and frequency of sessions. The interventions included aerobic, resistance and respiratory muscle training exercises. The results showed improvements in peak oxygen uptake, six minutes walking test, quality of life and inspiratory muscle strength, among others. We conclude that the evidence supports the recommendation of physical rehabilitation in selected patients with stable PAH as a complementary strategy to the available pharmacological therapy.

(Rev Med Chile 2018; 146: 627-635)

Key words: Exercise; Hypertension, Pulmonary; Rehabilitation.
'Escuela de Kinesiología, Facultad de Ciencias de la Salud, Universidad San Sebastián. Santiago, Chile.

2Dirección de Postgrado, Facultad de Ciencias, Universidad Mayor. Santiago, Chile.

${ }^{3}$ Escuela Kinesiología, Facultad de Ciencias de la Salud, Universidad de las Américas. Santiago, Chile.

${ }^{4}$ Escuela de Kinesiología, Facultad de Odontología y Salud, Universidad Diego Portales. Santiago, Chile.

5Programa de Fisiología y Biofísica, Instituto de Ciencias Biomédicas,

Facultad de Medicina, Universidad de Chile. Santiago, Chile.

${ }^{6}$ Departamento de Medicina. Facultad de Medicina. Universidad de Chile. Santiago, Chile.

'Instituto Nacional del Tórax. Santiago, Chile.

${ }^{8}$ Departamento de Enfermedades Respiratorias. Clínica Santa María. Santiago, Chile.

aKinesióloga.

bBioquímico.

CPhD.

Conflictos de interés: Ninguno que declarar. Este trabajo no obtuvo financiamiento.

Recibido el 14 de noviembre de 2017, aceptado el 14 de marzo de 2018.

Correspondencia a:

Mónica Zagolin

monizagolin@hotmail.com
L a hipertensión arterial pulmonar (HAP) grupo I de la Organización Mundial de la Salud (OMS) es una entidad crónica, progresiva y devastadora que afecta principalmente a mujeres jóvenes en plena edad productiva. Antes de la era farmacológica, la sobrevida era de 2,8 años ${ }^{1}$. Posterior a la década de 1990-99 se iniciaron las terapias específicas con los análogos de las prostaciclinas, luego, en el año 2002, fueron aprobados los inhibidores de receptores de endotelinas y, en el año 2005, los inhibidores de fosfodiesterasa 5. La última droga aprobada por la Food and Drug Administration (FDA) de los Estados Unidos de Norteamérica para uso en HAP es el riociguat (estimulador de la guanilato-ciclasa soluble), en el año $2013^{2,3}$. Hoy existe una amplia disponibilidad de medicamentos que actúan en las tres principales vías afectadas en esta patología (prostaciclinas, óxido nítrico y endotelinas). Las terapias específicas pueden ser usadas tanto en monoterapia como en modalidad combinada, la que puede ser implementada de manera conjunta desde el inicio de la terapia o bien de manera secuencial ${ }^{3}$. Pese a todo lo anterior, la sobrevida lograda es escasa aún y, según los registros europeos y americanos, no supera $65 \%$ a los 5 años $^{4,5}$. Para mejorar dicha sobrevida, posiblemente, se requiera de un mayor conocimiento sobre otras vías patogénicas o sobre la manera de enfrentar la terapia, además de un diagnóstico más precoz y un inicio oportuno de las terapias combinadas. Por otra parte, es fundamental asociar a la terapia farmacológica otras estrategias efectivas tendientes a mejorar la calidad de vida de los pacientes, permitiéndoles una adecuada reincorporación al mundo laboral, familiar y social. 
La rehabilitación cardiopulmonar ha demostrado ser beneficiosa en múltiples patologías cardiorrespiratorias crónicas, tales como insuficiencia cardíaca congestiva ${ }^{6}$, enfermedad pulmonar obstructiva crónica (EPOC) $)^{7}$ y fibrosis pulmonar ${ }^{8}$, entre otras. En la HAP existe información limitada sobre rehabilitación, proveniente de trabajos metodológicamente muy diferentes, que incluyen fundamentalmente población en capacidad funcional II-III, no existiendo información suficiente respecto a qué modalidad, frecuencia e intensidad de ejercicio se requieren para obtener los mejores resultados. Sin embargo, en el V simposio de expertos de Niza (2013) se aprobó la rehabilitación con indicación I-A, en atención a dos trabajos aleatorizados que mostraban beneficios significativos ${ }^{2}$. Posterior a aquello, se ha generado más información, tanto de efectividad como de seguridad, y las últimas guías europeas (2015) lo aprueban, aunque con una menor fuerza de evidencia y de recomendación (IIa-B), debido a la información faltante y a algunos aspectos de seguridad. En estas guías se deja muy en claro que la población blanco debe encontrarse estable y que la rehabilitación debe ser necesariamente supervisada ${ }^{3}$.

Si bien se requiere con urgencia más información respecto a cómo debe efectuarse la rehabilitación en estos pacientes, se han demostrado beneficios en lo que respecta a calidad de vida, capacidad funcional y rendimiento muscular (distancia recorrida en test de 6 min o tiempo para la fatiga). Sin perjuicio de lo anterior, no se han documentado beneficios en parámetros funcionales cardiorrespiratorios, tales como biomarcadores (péptido natriurético auricular, BNP o PRO-BNP) de función del ventrículo derecho (VD), indicadores ecográficos y hemodinámicos de disfunción de VD, y pruebas de función pulmonar9. Dado que la sobrevida en la HAP aún es reducida, pese a la mayor disponibilidad de terapias farmacológicas específicas, es que cobra particular relevancia su complementación con otras estrategias tendientes a modificar el curso natural de la enfermedad o, al menos, a optimizar la calidad de vida de estos pacientes. A lo anterior se suma el hecho de que estos pacientes principalmente se quejan de disnea con el esfuerzo, lo que se traduce en sedentarismo y atrofia, tanto muscular periférica como diafragmática, por lo cual una rehabilitación global -basada en el ejer- cicio- viene a cubrir la brecha no resuelta por la terapia farmacológica ${ }^{10}$.

A continuación, se detallan los principales resultados de las investigaciones publicadas respecto a la rehabilitación en HAP, realizándose un análisis crítico de la literatura, así como una recomendación acorde a los recursos y a las características de los pacientes en nuestro país.

\section{Metodología}

\section{Estrategia de búsqueda}

Se realizó una búsqueda computarizada de la literatura en las bases de datos MEDLINE, LILACS y COCHRANE, empleando los siguientes términos MeSH: Pulmonary arterial hypertension, rehabilitation, exercise training, physical training.

\section{Límites de la búsqueda}

Los límites de la estrategia de búsqueda fueron: estudios publicados en los últimos 12 años, con investigación en humanos, mayores de 18 años, de cualquier sexo o raza.

Los estudios debían estar en idioma inglés o español y ser de alguno de los siguientes tipos: clínicos aleatorizados o no aleatorizados, casos controles o series de casos.

\section{Criterios de inclusión}

El filtro de estudios se aplicó a los resúmenes en estudios clínicos aleatorizados o no aleatorizados, casos control y serie de casos, bajo las siguientes condiciones:

- Pacientes con HAP grupo I de la OMS: idiopática o asociada a otras entidades (enfermedades del tejido conectivo, cardiopatías congénitas, entre otras) en estudios en los que en el título hace referencia al entrenamiento físico.

- Estudios cuya intervención sea en más de una sesión de entrenamiento.

\section{Criterios de exclusión}

- Estudios piloto o en fase preliminar.

- Estudios de un caso.

- Estudios que utilizan un catéter arterial.

- Pacientes con otras patologías asociadas.

- Estudios sin conclusión o interpretación final de resultados.

- Estudios que no especifican el tipo de ejercicio aplicado y la secuencia o duración del mismo. 


\section{Resultados de la búsqueda}

Mediante la búsqueda en las bases de datos se encontró un total de 229 artículos, 201 de los cuales fueron eliminados al no reunir todos los criterios de inclusión. Esta selección de artículos dejó un total de 28 referencias potencialmente elegibles. Sin embargo, 13 de ellos fueron excluidos al aplicar los criterios de exclusión. Por lo tanto, para el análisis de la información se incluyeron los 15 artículos que cumplían con todos los requisitos antes descritos (Figura 1).

\section{Resultados}

Las características de todos los estudios analizados se detallan en la Tabla 1.

El artículo pionero en rehabilitación fue publicado en el año 2006 por el grupo alemán liderado por Mereles, empleando un grupo de treinta pacientes que fueron aleatoriamente distribuidos para realizar varias modalidades de ejercicio (ejercicios cardiorrespiratorios en bicicleta, caminata, pesas con mancuernas, elongación, técnicas respiratorias y yoga). El entrenamiento fue realizado por tres semanas en ambiente hospitalario y luego por doce semanas en domicilio, con un total de una hora y media al día, durante cinco veces por

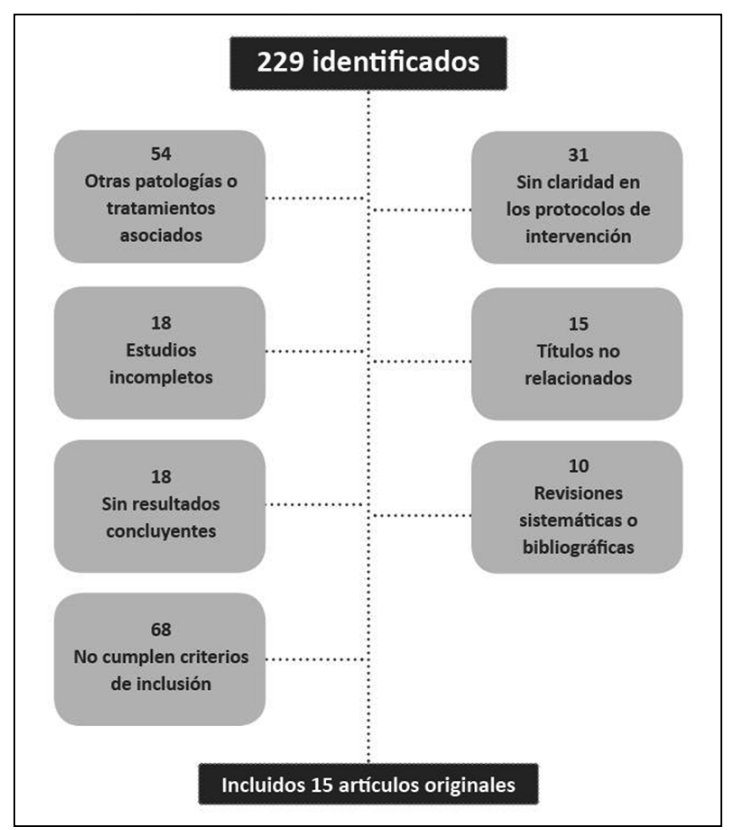

Figura 1. Diagrama de flujo para la selección de artículos. semana. El grupo presentó mejoría significativa en la distancia recorrida (DR) en caminata de 6 min y mejoría en el cuestionario de calidad de vida SF36, sumado a una mejoría en la clase funcional de la OMS (de I a IV, según la presencia de disnea, fatiga, dolor torácico o síncope), en el $\mathrm{VO}_{2}$ peak y en la carga máxima a tolerar en el test cardiopulmonar?.

Posteriormente, con protocolos similares, siguió el trabajo de De Man en el año 2009, quien demostró mejoría significativa en endurance (o tiempo a la fatiga) y aumento de la capilarización a nivel de miocitos ${ }^{11}$.

Al igual que en los precedentes, el trabajo de Fox $^{12}$, con 22 pacientes ejercitados por doce semanas en ambiente ambulatorio, mostró una mejoría significativa en la DR en el test de caminata de $6 \mathrm{~min}$, sin variaciones en parámetros hemodinámicos ecográficos ni en biomarcadores. Asimismo, el grupo de Grünig también demostró en varios ensayos clínicos no aleatorizados que la rehabilitación podría ser útil en otros grupos, como son los pacientes con cardiopatía congénita y enfermedades del tejido conectivo (ETC) ${ }^{14-16,20}$.

De los estudios publicados recientemente, es interesante destacar el artículo de González-Saiz ${ }^{22}$ y el de Santos-Lozano ${ }^{31}$, los que comienzan con un mismo ensayo clínico aleatorizado, en el que se entrenan a sujetos con HAP en un centro de salud por 8 semanas, demostrándose mejorías significativas en la $\mathrm{DR}, \mathrm{VO}_{2}$ peak y presión inspiratoria máxima (PImax). Lo más relevante de estas investigaciones es que por primera vez se continuó con un entrenamiento por 8 meses, describiéndose el efecto positivo sobre las variables estudiadas: prensa de piernas, prensa de banca, NT-proBNP, $\mathrm{VO}_{2}$ peak relativo, PImax y DR en test de marcha en $6 \mathrm{~min}$.

\section{Rehabilitación}

\section{Indicaciones}

La mayoría de los estudios efectúan la rehabilitación en ambiente hospitalario en una primera etapa de tres semanas de duración, seguidas por un programa ambulatorio de doce semanas (en promedio) y sin un protocolo estándar común ${ }^{9,14,17,20}$. Respecto a la monitorización de la rehabilitación en la práctica clínica habitual, en general, se sugiere que esta no sea invasiva ${ }^{9,14,17,20}$. 
Tabla 1. Resumen de las características principales de los estudios analizados

\begin{tabular}{|c|c|c|c|c|c|}
\hline $\begin{array}{l}\text { Autor/año } \\
\text { (referencia) }\end{array}$ & $\mathbf{n}$ & $\begin{array}{c}\text { Grupo } \\
\text { (subgrupo) }\end{array}$ & $\begin{array}{l}\text { ¿Aleatorizado? } \\
\text { (Sí/No) }\end{array}$ & $\begin{array}{l}\text { Desenlace } \\
\text { (Endpoint) }\end{array}$ & $\begin{array}{l}\text { Duración } \\
\text { rehabilitación } \\
\text { (semanas) }\end{array}$ \\
\hline Mereles/2006 9 & 30 & Idiopático & Sí & $\begin{array}{l}\text { VO }{ }_{2} \text { peak, DR, Carga } \\
\text { peak, CV }\end{array}$ & $\begin{array}{l}3 \text { en centro de salud } \\
9 \text { en casa }\end{array}$ \\
\hline De Man/2009'11 & 19 & Idiopático & No & $\begin{array}{l}\text { DR, Fuerza y resis- } \\
\text { tencia de cuádriceps. } \\
\text { Carga de trabajo a } \\
\text { Umbral anaeróbico }\end{array}$ & 12 en centro de salud \\
\hline Fox/2011 12 & 22 & Idiopático & No & DR & 12 en centro de salud \\
\hline Chan $/ 2013^{13}$ & 19 & Idiopático & Sí & $\begin{array}{l}\mathrm{DR} \text {, endurance, } \\
\mathrm{VO}_{2} \text { peak, carga }\end{array}$ & 10 en centro de salud \\
\hline Grunig/2011 14 & 58 & Idiopático & No & $\mathrm{DR}, \mathrm{VO}_{2}$ peak, CV & $\begin{array}{l}3 \text { en centro de salud } \\
12 \text { en casa }\end{array}$ \\
\hline Grunig/2012 15 & 21 & ETC & No & $\mathrm{DR}, \mathrm{CV}$ & $\begin{array}{l}3 \text { en centro de salud } \\
12 \text { en casa }\end{array}$ \\
\hline Grunig/2012 16 & 183 & Idiopático & No & $\mathrm{DR}, \mathrm{CV}$ & $\begin{array}{l}3 \text { en centro de salud } \\
12 \text { en casa }\end{array}$ \\
\hline Nagel/2012 ${ }^{17}$ & 35 & $\begin{array}{l}\text { CTEPH inoperable o } \\
\text { residual }\end{array}$ & No & $\begin{array}{l}\mathrm{DR}, \mathrm{CV}, \mathrm{VO}_{2} \text { peak } \\
\text { carga de trabajo }\end{array}$ & $\begin{array}{l}3 \text { centro salud y } \\
15 \text { en casa }\end{array}$ \\
\hline $\begin{array}{l}\text { Becker-Grunig/ } \\
2013^{18}\end{array}$ & 20 & $\begin{array}{l}\text { Secundario a enferme- } \\
\text { dad congénita cardiaca }\end{array}$ & No & DR & $\begin{array}{l}3 \text { en centro de salud y } \\
12 \text { casa }\end{array}$ \\
\hline Weinstein/2013 19 & 24 & $\begin{array}{l}\text { Idiopática (6), ETC mixta } \\
\text { (1), AR (1), escleroderma } \\
(12), \text { SS (2), lupus (2) }\end{array}$ & Sí & $\begin{array}{l}\text { Fatiga, nivel de activi- } \\
\text { dad física, DR }\end{array}$ & $\begin{array}{l}10 \text { con supervisión } \\
\text { médica }\end{array}$ \\
\hline Kabitz/2014 20 & 7 & Idiopático (5), APAH (2) & No & $\begin{array}{l}\text { Fuerza muscular respi- } \\
\text { ratoria, DR }\end{array}$ & $\begin{array}{l}3 \text { en centro de salud } \\
12 \text { en casa }\end{array}$ \\
\hline Raskin/2014²1 & 23 & Sin etiología reportada & No & DR, disnea & $\begin{array}{l}17 \text { a } 36 \text { sesiones ( } 2 \text { o } \\
3 \text { veces por semana en } \\
\text { centro de salud) }\end{array}$ \\
\hline $\begin{array}{l}\text { González- } \\
\text { Saiz/201722 }\end{array}$ & 40 & $\begin{array}{c}\text { Idiopático (36) o } \\
\text { CTEPH (4) }\end{array}$ & Sí & $\begin{array}{l}\text { Peak de potencia } \\
\text { muscular }\end{array}$ & 8 centro de salud \\
\hline $\begin{array}{l}\text { Santos- } \\
\text { Lozano/201731 }\end{array}$ & 40 & $\begin{array}{l}\text { Idiopático }(90 \%) \text { o } \\
\text { CTEPH }(10 \%)\end{array}$ & Sí & $\begin{array}{l}\text { Peak de potencia en } \\
\text { piernas y prensa de } \\
\text { banca }\end{array}$ & 8 meses \\
\hline Talwar/201732 & 18 & Sin etiología reportada & No & $\begin{array}{l}\text { Tolerancia al ejercicio } \\
\text { (duración y velocidad } \\
\text { alcanzada) }\end{array}$ & 12 centro de salud \\
\hline
\end{tabular}

DR: Distancia recorrida en test de caminata de 6 min; CV: calidad de vida; ETC: Enfermedad del tejido conectivo; AR: Artritis reumatoidea; SS: Síndrome de Sjögren; $\mathrm{VO}_{2}$ : Consumo de oxígeno; $\mathrm{VO}_{2}$ peak: Consumo de oxígeno peak; CPET: Test de ejercicio cardiopulmonar; CTEPH: Tromboembolismo crónico por hipertensión pulmonar; APAH: Hipertensión pulmonar arterial asociada.

La rehabilitación estaría indicada para todo paciente con HAP en clase funcional I a III, de curso clínico estable en los últimos 3 meses $^{3,9,14}$. Se sugiere efectuar de preferencia en ambiente ambulatorio por un mínimo de doce semanas con supervisión estricta, para luego continuar en for- ma teledirigida o ambulatoria (en domicilio o en centros especializados), con asistencia a distancia y controles permanentes ${ }^{3}$.

Los pacientes estables más de tres meses en clase funcional IV, esperando trasplante $\mathrm{u}$ otra estrategia terapéutica, también deberían ser ob- 
jeto de rehabilitación, aunque cada caso debe ser analizado individualmente y la rehabilitación -programada en forma individualizada y con estricta supervisión-debería ser realizada en centros especializados.

Otros grupos de HAP, como el grupo IV de la OMS con hipertensión pulmonar asociada a tromboembolismo pulmonar crónico, también podrían ser objeto de rehabilitación antes y después del procedimiento quirúrgico terapéutico para este grupo como es la endarterectomía pulmonar, aunque en este grupo, la experiencia en rehabilitación es escasa y es menos consistente, por lo que se recomienda una evaluación caso a caso ${ }^{3,14}$.

\section{Seguridad del entrenamiento físico}

En todos los ensayos revisados se establece que la implementación y ejecución de los programas de rehabilitación deben ser efectuados en un centro clínico con experiencia en HAP y en rehabilitación de pacientes crónicos, a fin de minimizar los riesgos del entrenamiento a través de la monitorización constante y trabajo guiado ${ }^{14-16}$. El monitoreo antes, durante y posterior al entrenamiento, es un elemento crucial, considerando la labilidad de estos pacientes y por ello se sugiere que sea supervisado por personal especializado. Sin embargo, son pocos los estudios que abordan este tópico de manera específica ${ }^{14,15}$. En la Tabla 2 se muestra que los efectos adversos son menores cuando el programa es bien supervisado. En el reporte de Grünig y colaboradores sobre seguridad efectuando un análisis de 183 pacientes con hipertensión pulmonar de diferentes etiologías, incluidos los con insuficiencia cardíaca izquierda, sometidos a 3 semanas de entrenamiento en hospital, se documenta $13 \%$ de efectos adversos asociados al entrenamiento. De ellos, cabe destacar que hubo 2 síncopes (1\%), ocurridos horas luego del ejercicio, no directamente relacionados con el ejercicio, ya que en un caso se presentó en conjunto con una infección respiratoria. Los otros eventos adversos serios fueron infecciones respiratorias en $7 \%$ de los pacientes y arritmias autolimitadas en $1 \%{ }^{16}$. Sin duda, se requiere mayor información sobre este aspecto, sin embargo, por el momento, siendo el procedimiento supervisado y monitorizado, se puede considerar seguro en pacientes estables aptos para ingresar a un programa de rehabilitación.

En el caso que el paciente esté bien estabilizado, se podrían dar recomendaciones de actividades físicas sencillas para el hogar, como lo son las caminatas, con el objeto de minimizar los riesgos. En tales casos, la supervisión se realiza a través de llamadas telefónicas por parte del médico tratante, o bien del fisioterapeuta a cargo ${ }^{24-26} \mathrm{y}$, probablemente, sea recomendable que los pacientes dispongan de un oxímetro de pulso en domicilio para limitar sus ejercicios evitando taquicardias $>120 /$ min y desaturaciones $<90 \%$.

\section{Protocolo entrenamiento}

Modalidad: tipo, duración y frecuencia del ejercicio

Si bien aún no existe consenso en el tipo de ejercicio a realizar, de acuerdo a lo observado en los trabajos publicados parece apropiado que los pacientes efectúen un calentamiento en bicicleta a cargas crecientes alternadas, partiendo con la carga equivalente a $50 \%$ de su $\mathrm{VO}_{2}$ peak, y monitoreando frecuencia cardíaca, saturación de oxígeno, frecuencia respiratoria y presión arterial ${ }^{14-16}$. De la misma manera, los estudios analizados promueven intensidades de trabajo entre 60 y $80 \%$ del $\mathrm{VO}_{2}$ peak, con incrementos en la intensidad del ejercicio que dependen de la tolerancia individual ${ }^{12,20}$. Es motivo de detención del ejercicio para reposo si la frecuencia cardíaca supera los 120/ min y la saturación cae a cifras inferiores a 90\%, de manera de permitir una reacomodación antes de continuar o apoyar con oxígeno suplementario, de ser necesario, para facilitar una rehabilitación más efectiva ${ }^{12,20}$.

No queda claro el papel que les cabe a la elongación y a los ejercicios de equilibrio, pero sin duda son un buen complemento, al igual que el entrenamiento de diafragma ${ }^{12-26}$. Asimismo, se recomienda que en los días en que no se realice entrenamiento aeróbico, se haga entrenamiento de musculatura respiratoria y ejercicios de carga por 15 a $30 \mathrm{~min}^{24-26}$. Por otra parte, falta evidencia que demuestre si una modalidad exclusivamente de cargas es igual o superior a un entrenamiento fundamentalmente aeróbico, al igual que determinar cuáles son las periodicidades y tiempos óptimos.

En cuanto a la duración del entrenamiento, existen algunos artículos que señalan que se puede lograr éxito en el tratamiento con un entrenamiento físico que logre una regularidad similar a la de la medicación recibida; es decir, debe ser continuo, por al menos $15 \mathrm{~min} /$ día durante 5 días/ 
Tabla 2. Principales resultados de los estudios analizados

\begin{tabular}{|c|c|c|c|}
\hline $\begin{array}{l}\text { Autor/año } \\
\text { (referencia) }\end{array}$ & Desenlace primario & Desenlace secundario & $\begin{array}{l}\text { Eventos } \\
\text { adversos } \\
\text { ( } n \text { de casos) }\end{array}$ \\
\hline Mereles/2006 9 & $\begin{array}{l}\text { Aumento en DR }(96 \mathrm{~m})(p<0,001) \\
\text { Mejora en CV SF36 }(p<0,005)\end{array}$ & $\begin{array}{l}\text { Aumento en umbral anaeróbico, } \mathrm{VO}_{2} \text { peak y } \\
\text { PAPS }+(p<0,05) \text {. Test } \mathrm{WHO}-\mathrm{Q} \text { oL: mejoras } \\
\text { en } 3 \text { semanas }(p=0,02) \text { y en } 15 \text { semanas } \\
\text { ( } p<0,001) \text { : Parámetros mentales }(p=0,027) \text {, } \\
\text { funcionalidad social }(p=0,002) \text {, salud mental } \\
(p=0,017) \text { y vitalidad }(p<0,001)\end{array}$ & Ninguno \\
\hline $\begin{array}{l}\text { De Man/ } \\
2009^{11}\end{array}$ & $\begin{array}{l}\text { Aumentos en } \mathrm{DR}(4 \%)(p=0,13) \text {. Car- } \\
\text { ga de trabajo umbral anaeróbico ( } 32 \text { a } \\
46 \text { watts; } p=0,003) \text {. }\end{array}$ & $\begin{array}{l}\text { La resistencia de cuádriceps y la fuerza, } \\
\text { mejoraron } 13 \% \text { y } 34 \% \text {, respectivamente } \\
(p<0,05)\end{array}$ & Leve mareo (2) \\
\hline Fox/201112 & Aumento en DR $(32 \mathrm{~m})(\mathrm{p}<0,05)$ & $\mathrm{VO}_{2}$ peak mejora $(\mathrm{p}<0,05)$ & Ninguno \\
\hline Chan/2013 ${ }^{13}$ & Aumento en DR $(56 \mathrm{~m})(p=0,002)$ & Mejora en test CV SF36 $(p<0,05)$ & Ninguno \\
\hline Grünig/2011 14 & $\begin{array}{l}\text { Aumento en DR }(87 \mathrm{~m})(\mathrm{p}<0,001) \mathrm{y} \\
\mathrm{VO}_{2} \text { peak }(2,1 \mathrm{ml} / \mathrm{kg} / \mathrm{min})(\mathrm{p}<0,001)\end{array}$ & Mejora en test CV SF36 $(p<0,05)$ & $\begin{array}{l}\text { Mareo con el en- } \\
\text { trenamiento }(2)\end{array}$ \\
\hline Grünig/2012 15 & $\begin{array}{l}\text { Aumento en DR ( } 67 \mathrm{~m} \text { a las } 3 \text { semanas } \\
\text { y } 71 \mathrm{~m} \text { a las } 15 \text { semanas) }(p<0,05)\end{array}$ & Mejora en test CV SF36 $(p<0,05)$ & $\begin{array}{l}\text { Infección gastroin- } \\
\text { testinal (1), infec- } \\
\text { ción respiratoria (2) }\end{array}$ \\
\hline Grünig/2012 $2^{16}$ & $\begin{array}{l}\text { Aumento en DR ( } 68 \mathrm{~m} \text { a las } 3 \text { semanas } \\
\text { y } 78 \mathrm{~m} \text { a las } 15 \text { semanas) }(p<0,001)\end{array}$ & Mejora en test CV SF36 $(p<0,05)$ & $\begin{array}{l}\text { Presíncope post } \\
\text { entrenamiento (1), } \\
\text { Taquicardia durante } \\
\text { entrenamiento (2) }\end{array}$ \\
\hline Nagel $/ 2012^{17}$ & $\begin{array}{l}\text { Aumento en DR ( } 61 \mathrm{~m} \text { a las } 3 \text { semanas } \\
\text { y } 71 \mathrm{~m} \text { a las } 15 \text { semanas) }(p<0,001)\end{array}$ & $\begin{array}{l}\text { Mejoras en test CV SF36 }(p<0,05) \text { y niveles } \\
\text { de NT-ProBNP }(-20 \%) \text {. Sobrevida a los } 1,2 \\
\text { y } 3 \text { años fue de } 97 \%, 94 \%, 86 \% \text {, respecti- } \\
\text { vamente }\end{array}$ & $\begin{array}{l}\text { Síncope (1). } \\
\text { Herpes zoster (1) }\end{array}$ \\
\hline $\begin{array}{l}\text { Becker-Grünig/ } \\
2013^{18}\end{array}$ & $\begin{array}{l}\text { Aumento en DR ( } 63 \mathrm{~m} \text { a las } 3 \text { semanas } \\
\text { y } 67 \mathrm{~m} \text { a las } 15 \text { semanas })(p<0,001) \text {. } \\
\text { Incremento en } \mathrm{VO}_{2} \text { peak }(0,72 \mathrm{~L} / \mathrm{min} \\
\text { a las } 3 \text { semanas y } 0,95 \mathrm{~L} / \mathrm{min} \text { a las } 15 \\
\text { semanas) }(p=0,002)\end{array}$ & $\begin{array}{l}\text { Mejora en test CV SF36 ( } p<0,05) \text {. La } \\
\text { sobrevida en } 2 \text { años fue de } 100 \% \text {, y en } \\
\text { trasplantados } 100 \% \text { al primer año y } 93 \% \text { al } \\
\text { segundo año }\end{array}$ & Ninguno \\
\hline $\begin{array}{l}\text { Weinstein/ } \\
2013^{19}\end{array}$ & Aumento en DR $(53,5 \mathrm{~m})(p=0,003)$ & $\begin{array}{l}\text { Aumento en el peak de potencia de piernas } \\
(p=0,003)\end{array}$ & Ninguno \\
\hline Kabitz/2014 20 & $\begin{array}{l}\text { Aumento en DR ( } 92 \mathrm{~m} \text { a las } 3 \text { semanas } \\
\text { y } 81 \mathrm{~m} \text { a las } 15 \text { semanas) }(p<0,001) \text {. } \\
\text { Aumentos en PImax y PEmax, posterior } \\
\text { a } 15 \text { semanas }(p=0,086 \text { y } p=0,021 \text {, } \\
\text { respectivamente) }\end{array}$ & $\begin{array}{l}\text { Mejora en SnPna posterior a las } 15 \text { semanas } \\
(p=0,025)\end{array}$ & Ninguno \\
\hline Raskin/2014 21 & $\begin{array}{l}\text { Aumento en DR ( } 86 \text { m y } 53 \text { m para pa- } \\
\text { cientes que caminaban menos o más } \\
\text { de } 250 \mathrm{~m} \text { al inicio, respectivamente) } \\
(p=0,0012)\end{array}$ & $\begin{array}{l}\text { Cuestionario SGRQ muestra mejora en el } \\
\text { ámbito del impacto (de } 37,9 \text { a } 33,6,4,3) \text { y } \\
\text { un descenso en el dominio de actividad }(69,1 \\
\text { a } 73,4,4,3)^{*}\end{array}$ & Ninguno \\
\hline $\begin{array}{l}\text { González-Saiz/ } \\
2017^{22}\end{array}$ & $\begin{array}{l}\text { Mejora del peak de fuerza grupo con } \\
\text { ejercicio: Prensa de piernas (+166 } \\
\text { Watts) y prensa de brazos (+53 watts) }\end{array}$ & $\begin{array}{l}\text { Mejoras en DR, PIMax, VO }{ }_{2} \text { peak }(p<0,001) \\
\text { Cambios en NT-proBNP }(p g / m)(N S)\end{array}$ & Ninguno \\
\hline $\begin{array}{l}\text { Santos-Lozano/ } \\
2017^{31}\end{array}$ & $\begin{array}{l}\text { Mejora peak de fuerza grupo con } \\
\text { ejercicio: Prensa de piernas y prensa } \\
\text { de brazos }\end{array}$ & $\begin{array}{l}\text { NT-proBNP aumento en grupo con ejercicio } \\
\text { vs control.VO } \mathrm{O}_{2} \text { peak relativo, mejora grupo } \\
\text { con ejercicio PImax y } 6 \mathrm{MWT} \text { mejora en grupo } \\
\text { con ejercicio }\end{array}$ & Ninguno \\
\hline Talwar/201732 & $\begin{array}{l}\text { TE: Mejora en grupo con ejercicio } \\
(p<0,0001)\end{array}$ & No reportado & Ninguno \\
\hline
\end{tabular}


semana ${ }^{19,24}$. Sin embargo, otros estudios indican que una duración promedio de 1,5 h/día de entrenamiento es necesaria para lograr los efectos óptimos en estos pacientes ${ }^{9,12,14}$.

En términos prácticos, el entrenamiento físico se puede realizar en una bicicleta ergométrica o en una trotadora, donde el paciente alcance la frecuencia cardíaca indicada al menos 5 días a la semana $^{20,21}$.

\section{Entrenamiento en intervalos de alta intensidad (HIIT)}

En los últimos años se ha visto como el entrenamiento tipo HIIT (High Intensity Interval Training) ha logrado beneficios en distintos grupos de pacientes con patologías cardiorespiratorias ${ }^{27-29}$.

En lo que respecta a HAP, a la fecha solo se han investigado los efectos del HIIT en modelos animales, destacando el estudio realizado por Brown $\mathrm{MB}$ en ratas con HAP leve ${ }^{30}$. En dicho trabajo, se investigó la respuesta al HIIT en comparación con el ejercicio aeróbico continuo, durante 6 semanas de entrenamiento. Con ambos tipos de ejercicio mejoró la capacidad aeróbica máxima, pero solo en el grupo de ratas entrenadas con HIIT se redujo la presión sistólica del ventrículo derecho, la hipertrofia ventricular izquierda y la resistencia pulmonar total, aumentando el inotropismo del ventrículo izquierdo y la hemodinamia.

Si bien los resultados en animales son alentadores, no es fácil extrapolar su éxito en los humanos. Lógicamente, el riesgo inherente a este tipo de entrenamiento dificulta la realización de estudios similares en humanos. Sin perjuicio de lo anterior, resulta necesario explorar los beneficios del HIIT en pacientes con HAP, lo que sin duda requerirá tomar todos los resguardos necesarios. Una vez que dichos obstáculos sean salvados, y aunque el avance sea pausado, es posible que esta área concentre en el futuro el desarrollo más promisorio para el abordaje de la HAP mediante el ejercicio terapéutico.

\section{Conclusión}

La rehabilitación cardiorrespiratoria en los pacientes con HAP ha demostrado ser parte relevante del tratamiento y control de esta patología, como se desprende de los artículos incluidos en esta revisión. Los estudios demuestran mejoras significativas en diversos parámetros fisiológicos en dichos pacientes, independientes de su clase funcional, al ser sometidos a programas de entrenamiento físico estructurados. De particular relevancia es el hecho de que la mayoría de los estudios demuestra una mejoría en la tolerancia al ejercicio, en las actividades de la vida diaria y en la calidad de vida.

$\mathrm{Si}$ bien es cierto que los trabajos hasta ahora realizados constituyen aún la antesala para ensayos clínicos más exhaustivos (que puedan medir parámetros funcionales, hemodinámicos y biomoleculares involucrados en el curso natural de la enfermedad, su progresión y deterioro), no es menos cierto que resultan promisorios a la hora de evaluar el impacto del ejercicio terapéutico en la HAP.

En síntesis, la evidencia disponible apoya la recomendación de rehabilitación en los pacientes con HAP como una estrategia complementaria a la terapia farmacológica disponible, constituyéndose así como un pilar adicional en el abordaje terapéutico integral de esta enfermedad.

\section{Referencias}

1. D’Alonzo GE, Barst RJ, Ayres SM, Bergofsky EH, Brundage BH, Detre KM, et al. Survival in patients with primary pulmonary hypertension. Results From a national prospective registry. Ann Intern Med 1991; 115 (5): 343-9.

2. Galiè N, Corris P, Frost A, Gigis R, Granton J, ChengJing, et al. Updated Treatment Algorithm of pulmonary arterial hypertension. J Am Coll Cardiol 2013; 62 (25): 60-72.

3. Galiè N, Humbert M, Vachiery JL, Gibbs S, Lang I, Torbicki A, et al. The 2015 ESC/ERS guidelines for the diagnosis and treatment of pulmonary y hypertension. Eur Respir J 2015; 46: 879-82.

4. Benza RL, Miller DP, Gomberg-Maitland M, Frantz RP, Foreman AJ, Coffey CS, et al. Predicting Survival in pulmonary arterial hypertension: insights from Registry to Evaluate Early and Long-Term Pulmonary Arterial Hypertension Disease Management (REVEAL).Circulation 2010; 122: 164-72.

5. Humbert M, Sitbon O, Chaouat A, Bertocchi M, Habib $\mathrm{G}$, Gressin V, et al. Survival in patients with idiopathic, familial, and anorexigen-associated pulmonary arterial hypertension in the modern management era. Circulation 2010; 122 (2): 156-63. 
6. Servey JT, Stephens M. Cardiac Rehabilitation: Improving Function and Reducing Risk. Am Fam Physician 2016; 94 (1): 37-43.

7. Casaburi R, ZuWallack R. Pulmonary rehabilitation for management of chronic obstructive pulmonary disease. N Engl J Med 2009; 360 (13): 1329-35.

8. Vainshelboim B, Fox BD, Oliveira J, Kramer MR. Exercise training in idiopathic pulmonary fibrosis. Expert Rev Respir Med 2016; 10 (1): 69-77.

9. Mereles D, Ehlken N, Kreuscher S, Ghofrani S, Hoeper M, Halank M, et al. Exercise and respiratory training improve exercise capacity and quality of life in patients with severe chronic pulmonary hypertension. Circulation 2006; 114: 1482-9.

10. Zagolin BM, Wainstein GE, Uriarte GP. [Update in the diagnosis and therapy for pulmonary arterial hypertension]. Rev Med Chile 2006; 134 (7): 902-9.

11. De Man FS, Handoki ML, Groepenhoff H, van't Hui AJ, Koppers A, Grotjohan HP, et al. Effect of exercise traininig in patients with idiopathic pulmonary arterial hypertension. Eur Respir J 2009; 34: 669-75.

12. Fox B, Kassirer M, Weiss I, Raviv Y, Peled N, Shitrit D et al. Ambulatory Rehabilitacion Exercise Capacity in patients with pulmonary hypertension. J Card Fail 2011; 17: 106-200.

13. Chan K, Chin L, Kennedy M, Woolstenhulme J, Nathan S, Weinstin A, et al. Benefits of intensive treadmill exercise training cardiorespiratory function and quality of life in patients with pulmonary hypertension. Chest 2013; 143 (2): 333-43.

14. Grünig E, Ehlken N, Ghofrani A, Staehler G, Meyer FJ, Juenger J, et al. Effect of exercise and respiratory training on clinical progression and survival in patients with severe chronic pulmonary hypertension. Respiration 2011; 81: 394-401.

15. Grünig E, Maier F, Ehlken N, Fisher C, Lichtlau M, Blank $\mathrm{N}$, et al. Exercise training in pulmonary arterial hypertension associated with connective tissue diseases. Arthritis Res Ther 2012; 14: R148.

16. Grünig E, Lichtblau M, Ehlken N, Ghofrani HA, Reichenberg F, Staehler G, et al. Safety and efficacy of exercise training in various forms of pulmonary hypertension. Eur Respir J 2012; 40: 84-92.

17. Nagel C, Prange F, Guth S, Herb J, Ehlken N, Fisher C, et al. Exercise training improves exercise capacity and quality of life in patients with inoperable or residual chronic thromboembolic pulmonary hypertension. PLoSOne 2012; 7: e41603.

18. Becker-Grünig T, Klose H, Ehlken N, Lichtblau M, Nagel C, Fischer C, et al. Efficacy of exercise training in pulmonary arterial hypertension associated with congenital heart disease. Int J Cardiol 2013; 168 (1): 375-81.

19. Weinstein AA, Chin LM, Keyser RE, Kennedy M, Nathan SD, Woolstenhulme JG, Connors G, et al. Effect of aerobic exercise training on fatigue and physical activity in patients with pulmonary arterial hypertension. Respir Med 2013; 107: 778-84

20. Kabitz HJ, Bremer HC, Schwoerer A, Sonntag F, Walterspacher S, Walker DJ, et al. The Combination of exercise and respiratory training improves respiratory muscle function in pulmonary hypertension. Lung 2014; 192: 321-8.

21. Raskin J, Qua D, Marks T, Sulica R. A retrospective study on the effects of pulmonary rehabilitation in patients with pulmonary hypertension. Chron Respir Dis 2014; 11: 153-62.

22. González L, Fiuza C, Sanchis F, Santos A, Quezada CA, Flox A, et al. Benefits of skeletal-muscle exercise training in pulmonary arterial hypertension: The WHOLEi+12 trial. Int J Cardiol 2017; 15231: 277-83.

23. Rich S, Dantzker DR, Ayres SM, Bergofsky EH, Brundage $\mathrm{BH}$, et al. Primary Pulmonary Hypertension, a national prospective study. Ann Intern Med 1987; 107: 216-23.

24. Marra AM, Egenlau FB, Bossone E, Eichtaedt C, Grunig E, Ehlken N. Principles of Rehabilitation and Reactivation: Pulmonary Hypertension. Respiration 2015; 89: 265-73.

25. Ehlken N, Verduyn C, Tiede H, Staehler G, Karger G, Nechwatal R, et al. Economic Evaluation of exercise training in patients with pulmonary hypertension. Lung 2014; 192: 321-8.

26. Arena R, Cahalin L, Borghi-Silva A, Myers J. The Effect of exercise training on the pulmonary arterial system in patients with pulmonary hypertension. Prog Cardiovasc Dis 2015; 57: 480-8.

27. Karlsen T, Aamot IL, Haykowsky M, Rognmo O. High, Intensity Interval Training for Maximizing Health Outcomes. Prog Cardiovasc Dis 2017; 17: 30051-58.

28. McGregor G, Nichols S, Hamborg T, Bryning L, Tudor-Edwards R, Markland D, et al. High-intensity interval training versus moderate-intensity steady-state training in UK cardiac rehabilitation programmes (HIIT or MISS UK): study protocol for a multicentre randomised controlled trial and economic evaluation. BMJ Open 2016; Article ID: e012843.

29. Cardozo GG, Oliveira RB, Farinatti PT. Effects of high intensity interval versus moderate continuous training on markers of ventilatory and cardiac efficiency in coronary heart disease patients. Scientific World Journal 2015; Article ID 192479. 
30. Brown MB, Neves E, Long G, Graber J, Gladish B, Wiseman A, et al. High-intensity interval training, but not continuous training, reverses right ventricular hypertrophy and dysfunction in a rat model of pulmonary hypertension. Am J Physiol Regul Integr Comp Physiol 2017; 312: 197-210.

31. Santos-Lozano A, Sanz-Ayan P, González-Saiz L, Quezada-Loaiza CA, Fiuza-Luces C, Flox-Camacho A, et al.
Effects of an 8-month exercise intervention on physical capacity, NT-proBNP, physical activity levels and quality of life data in patients with pulmonary arterial hypertension by NYHA class. Data Brief 2017; 12: 37-41.

32. Talwar A, Sonu Sahni S, Verma S, Sara Z. Khan, Sean Dhar, Nina Kohn. Exercise tolerance improves after pulmonary rehabilitation in pulmonary hypertension patients. J of Exerc Rehabil 2017; 13 (2): 214-7. 